\title{
Cross-cultural adaptation in French and validation of Functional Assessment Scale for acute Hamstring injuries (FASH)
}

\section{Sports}

\author{
M. Loquet ${ }^{4}$, T Willems², C Specque ${ }^{3}$, J. Van Beveren³ ${ }^{3}$ O. Bruyère ${ }^{4}$, JF. Kaux ${ }^{1,2}$
}

FIFA

MEDICAL
CENTRE OF
EXCELLENCE

1. Department of Public Health, Epidemiology and Health Economics, University of Liège, Liège, Belgium.

2. Physical Medicine and Sports Traumatology Department, University and University Hospital of Liège, Liège, Belgium.

Introduction: Acute hamstring injury is a very common muscle injury particularly in sports that require high explosive strength, sports with impulsions and sports with running phases. Among those sports, the most widespread are soccer, rugby and sprinting. The Functional Assessment Scale for acute Hamstring injuries (FASH) assesses pain, physical activity and how patients realize some exercises while suffering hamstring injury. Current available versons are in English, in German and in Greek.

Objectives: The aims of thus study were to reach a reliable and valid translation and a cross-cultural adaptation of this questionnaire in French.

Material and methods: FASH translation and adaptation were realized in respect of international guidelines. The population composed of 116 subjects filled the questionnaire twice separated by 48-60th. FASH reliability and validity were assessed thanks to SF-36 allowing us to assess the test retest reliability (with intra-class correlation ICC), internal consistency (with Cronbach's alpha coefficient and construct validity, with Spearman's correlation coefficients).

FASH-Fr: Échelle d'évaluation fonctionnelle pour blessures aigües des ischio-jambiers

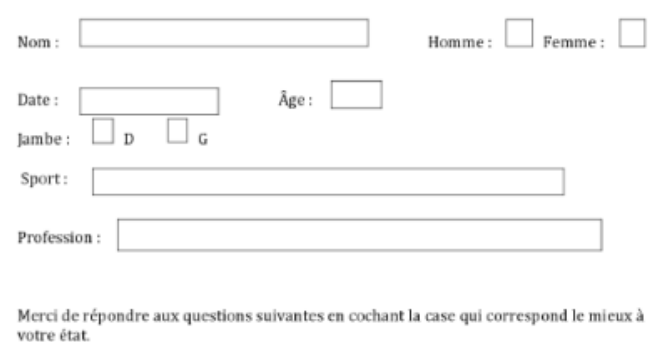

\section{FASH-Fr Questionnaire}

1. Si vous avez eu une lésion aigủe des ischio-jambiers, veuillez évaluer votre douleur et/ou votre gêne actuelle(s) :

$\begin{aligned} & \text { Douleur Insoutenable/ } \\ & \text { Symptômes }\end{aligned}$
$\begin{aligned} & \text { 2. Actuellement, pratiquez-vous votre activité sportive, votre } \\ & \text { entrainement ou toute autre activité physique ? }\end{aligned}$
[ Non, plus du tout
国 Programme d'entrainement adapté
可 Niveau d'entrainement convenable mais différent d'avant la lésion
10 le peux m'entrainer/ participer à des activités physiques comme auparavant
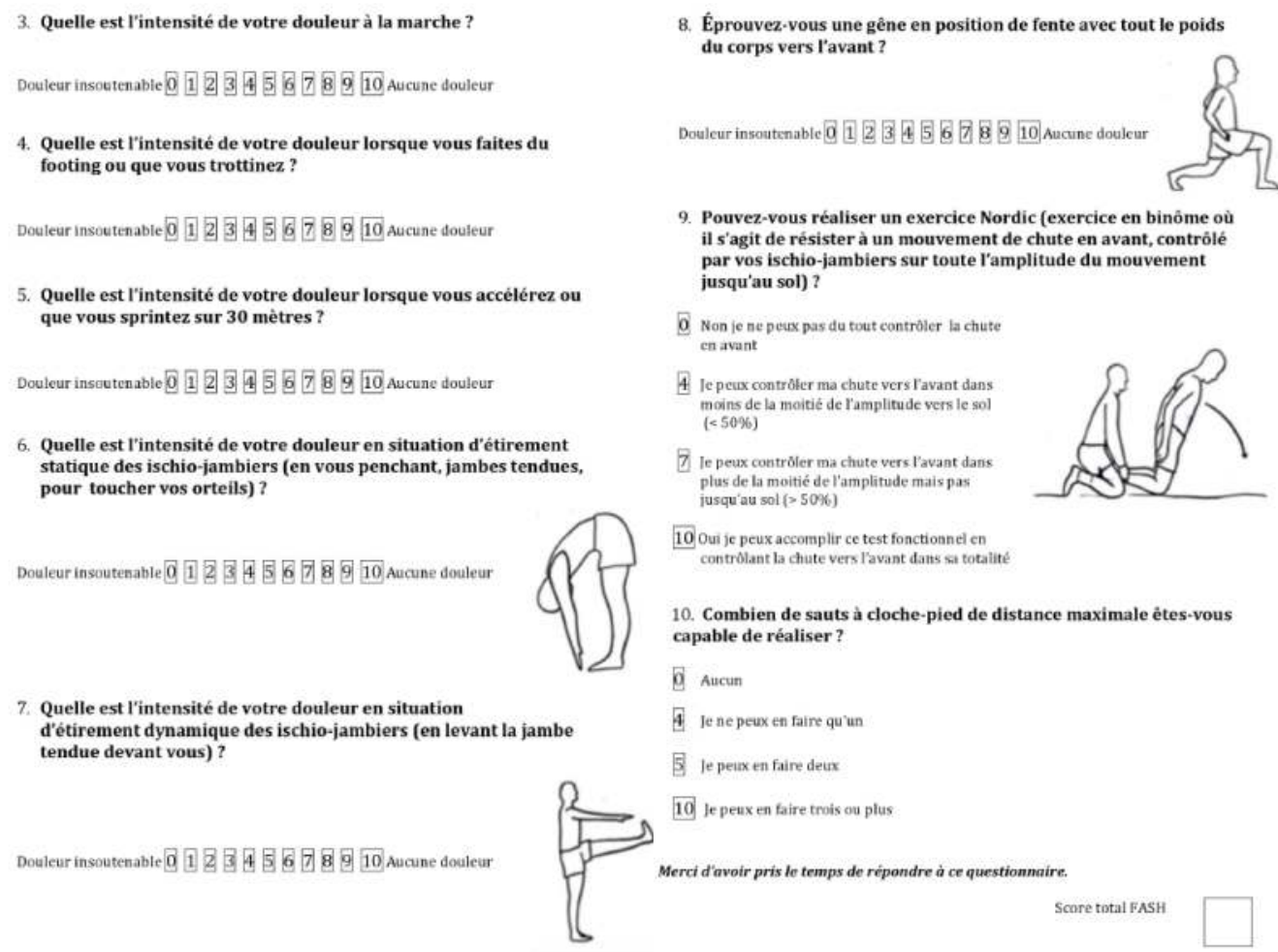
Eposition de fente avec tout le poids

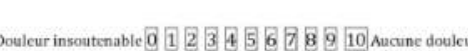

Pouvez-vous réaliser un exercice Nordic (exercice en binôme ou parte jusquau sol) ?

Non je ne peux pas du tout controler la chute

le peux controfler mà chute vers Favant dans noins de la moitie de ramplitude vers le si e peux contrôler ma chute vers lavant dos plus de' la moitié ée l'amplitude mais pas
jusqua sol ( $>50 \%$ )

Oui je peux aceomplir ce test fonctionnel cn 0. Combien de sauts à cloche-pied de distance maximale êtes-vous capable de réaliser?

0. Aucun

4 Je ne peux en faire qu'un

5. le perix en faire deux

d'étirement dynamique des ischio-jambiers (en levant la jam tendue devant vous) ?

Score total FAS

\section{Results}

ICC showed a strong correlation between test and re-test (ICC total=0.9985). Internal consistency was also found to be strong with Cronbach's alpha coefficient $=0.98$. Correlation between FASH and SF-36 overall score was considered as strong (Rs=0.69, $p<0.0001)$. Correlation with SF-36 sub scales ( $P F, R P, B P$ ) were also considered as strong, showing their convergent validity. Other SF-36 subclass $(\mathrm{GH}, \mathrm{MH}, \mathrm{REm}, \mathrm{SF}, \mathrm{VT})$ displayed a moderate correlation with FASH questionnaire, showing their divergent validity. No floor or ceiling effect was observed.

\section{Conclusion}

FASH translation and cross-cultural adaptation into French can be considered as successful. FASF-F is now a reliable and valid tool for patients suffering acute hamstring injury. 